\title{
Effect of conflict between the body parts on perceptual adaptation
}

\author{
GARY P. MANDELL and CARL $\hat{A}_{\mathbf{A}}$ UERBACH \\ Yeshiva University, 55 Fifth Avenue, New York, New York 1003
}

\begin{abstract}
Two groups of subjects adapted to prismatic displacement. One adapted in a visual target condition, in which they pointed to an external visual target with their right index fingers. The other adapted in a body target condition, in which they pointed to their left index fingers with their right index fingers. The visual target group showed more adaptation of the right hand and less adaptation of the left hand than did the body target group. These results are consistent with an assimilation tendency within the proprioceptive modality, namely that body parts that are in contact tend to be perceived as occupying the same spatial position.
\end{abstract}

When a person wears prisms which displace the visual stimulus, he learns to adapt to the displacement. that is, to correct his initially maladaptive behavior. One of the conditions necessary for this adaptation is cue conflict, i.e., a discrepancy between spatial information concerning the same stimulus coming from different modalities (Moulden, 1971; Wallach, 1968). At present, it appears that adaptation to displaced visual stimulation is sometimes a linear sum of visual and proprioceptive changes and, at other times, includes higher order changes involving the correspondence rule between visual and proprioceptive information which registers the two sources of information as coincident. This latter change has sometimes been called a sensorimotor change (Hardt, Held, \& Steinbach, 1971) and sometimes called a change in coordinators (Templeton. Howard, \& Wilkinson, 1974). Whether the adaptive change is visual, proprioceptive, or a change in the coordination between them, the upshot of the adaptation is that sources of spatial information concerning the same object which previously differed come to agree. Wallach calls this change "process assimilation," and for brevity we will use the term "assimilation" in this paper to refer to it.

Most studies to date, however, have examined conflict and assimilation only between vision and proprioception. For example, when a subject points to a visual target while wearing prisms, there are two sources of conflict: (1) between the position of the target indicated visually and the position of the target indicated by proprioceptive information from the hand that touches it, and (2) between the position of

Requests for reprints should be sent to Dr. Carl Auerbach. Department of Psychology, Yeshiva University, 55 Fifth Avenue, New York, New York 10003. The authors are grateful to Dr. Ian Howard for his comments on the manuseript, and in particular for raising certain questions of interpretation which we have tried to answer. Preparation of this manuscript was partly supported by NSF Grant GB 44234 to the second author. the hand itself, indicated by proprioceptive information from the hand, and the position of the hand indicated by visual information concerning the hand.

The aim of the present study is to examine conflict and assimilation within the proprioceptive modality. Consider, for example, what happens when the right and left hands touch each other. In addition to proprioception from each hand indicating the position of that hand, each hand also provides information about the other hand. For example, if the left hand is located straight ahead of the body and is also touching the right hand, then the right hand must also be located straight ahead of the body. If the right hand would separately adapt so that it feels, say, $10 \mathrm{deg}$ to the right of the body when it is in fact straight ahead, then a conflict between the left and right hands would exist. since the left hand would indicate that it and the right hand were straight ahead whereas the right hand would indicate that it and the left hand were $10 \mathrm{deg}$ right. If the same assimilation tendency exists between proprioception of the right and left hands as exists between vision and proprioception, then, unless the right hand totally dominates the left or vice versa, one would expect the apparent spatial positions of the right and left hands to shift towards each other. The change could involve either proprioception of the right and left hands or "coordinators" between left- and right-hand proprioception. In either event, the effect of such change would be to reduce the adaptation of the right hand and, of course, to increase the adaptation of the left hand.

If. correspondingly, there were an impetus to adaptation to the right hand but not to the left hand, but the contact between them was maintained, then the hypothesized assimilation tendency would act so as to reduce the effect of the adaptation conditions below what it would be if the contact was not present. Similarly, even without any direct impetus to adaptation to the left hand, the effect of the contact 
"ould be to produce some adaptation in the left hand.

In order to examine this hypothesized assimilation wheney whin the proprieceptive modality, we used atl experimental situation in which subjects pointed to a part of their own bodies with the right index tinger. The body part to which they pointed was the index finger of the leti hand. The left hand and arm were entirely visible to the subjects. except for the letit index tinger, which pointed downward through a hole. The pointing movement involved reaching to the concealed lett index finger with a ballistic movement of the right hiand. In order to aroid hopelessly contounding the situation. the only feedback available was when the tinger was actually touched. so that the subjects received no visual information about their errors. The condition just mentioned will be called the body target condition. In a control condition, the visual target condition, the left index finger. as target, was replaced by a wooden dowel occupying the same position as the index finger.

The body target condition induces two sorts of contlict in the right hand. namely: (1) between the position of the right hand indicated by proprioception from the right hand and the position of the right hand as indicated by visual information concerning the target which it touches, and (2) between the position of the right hand as indicated by proprioception from the right hand and the position of the right hand as indicated by proprioception from the left hand. Only the first conflict is present in the visual target condition.

In the visual target condition, there is an assimilation tendency between vision and proprioception which would promote adaptation of the right hand. In the body target condition, the same assimilation tendency exists, but there is also an opposing assimilation tendency due to proprioceptive information from the left hand, namely that which was discussed earlier. To the degree that left-hand proprioception remains veridical in the body target condition, it serves to limit right-hand adaptation through the mechanism of this opposing assimilation tendency. Carrying this reasoning one step further, we would hypothesize that to the degree that left-hand adaptation is complete, right-hand adaptation would not differ in the visual target and the body target conditions. However, since we neither anticipated nor found left-hand proprioceptive adaptation to be complete, this situation did not arise. Consequently, it is predicted that there will be less adaptation of the right hand in the body target condition than in the visual target condition.

In addition, a prediction is made concerning adaptation in the left hand. In the body target condition, there are two sources of conflict involving the left hand: (1) between visual information about the left hand and proprioceptive information from the left hand. (2) between proprioceptive information from the left hand concerning its own position, and proprioceptive information from the right hand concerning the position of the lett hand. Each of these conflicts has a separate assimilation tendency. The tirst would produce an adaptive shift in the left hand, and the second would oppose such a shift. At present, we cannot predict the exact resolution of this conflict between assimilation tendencies, except to say that there will be less adaptation when it is present than when it is absent. However, there will still be some adaptation. due to contact between the pointing right hand and the pointed-to left hand. Since there is no reason to expect genuine adaptive changes for the lett hand. i.e., nonvisual changes, more adaptation for the leti hand is predicted in the body target condition than in the visual target condition.

\section{METHOD}

\section{Apparatus}

The subjects were seated in front of a $76.2-\mathrm{cm}$-high table, on which rested a $60.04-\mathrm{cm}$ square frame, which was supported by four $20.32-\mathrm{cm}$ legs. A bite board was centered on the frame, and subjects were instructed to bite on the board. which had the effect of restricting head morements.

The entire frame was covered by a wooden top which prevented the subject from secing his pointing arm. Direetly in front of the subject and $43.18 \mathrm{~cm}$ away was a hole through which a $1.91-\mathrm{cm}-\mathrm{diam}, 35.50 \cdot \mathrm{cm}-\mathrm{long}$ wooden dowel could be inserted. When it was inserted, it extended vertically from the table top to a point $12.7 \mathrm{~cm}$ above the surface of the frame.

fin the body target condition, the subject rested his left hand over the trame top and insered his left index tinger through the hole. In the visual target condition, the dowel was inserted through the hole.

\section{Procedure and Design}

The experiment was run in three parts: a pretest of pointing straight ahead and of pointing to a visual target without feedback: an exposure period in which the two groups of subjects pointed to the targets appropriate to the group; and a posttest. also of pointing straight ahead and pointing at a visual target without feedback.

Pretest. The subject was seated in front of the apparatus with his eyes closed: he then put on 20 diopter base-left prisms. The prisms were mounted in a wire eyeglass frame with the left eye occluded. The subject wore goggles over the prisms which restricted his periptueral vision. In order to measure pretest pointing at a visual target. the subject was asked to point to the visual target five times with the left index finger and tive times with the right index finger. In order to meatsure pretest straight-ahead pointing, the subject was asked to point straight ahead with his eves closed. five times with the index finger of the left hand and five times with the index finger of the right hand. A barrier was inserted during the pretest which prevented the subject from actually touching the target. In each calse. the direction in which the subject pointed was recorded by the experimenter. Pointing to the visual target and pointing straight ahead were counterbalanced, as were pointing with the right and lift hands. The subject was instructed to point with a ballistic movement upon receiving the experimenter's signal, and then to return his hand to a resting position on the table in front of him. The signals to point were given every $15 \mathrm{sec}$.

Exposure. The exposure period consisted of $3 \mathrm{~min}$ of target pointing, wearing prisms. once every $15 \mathrm{sec}$. After the pretest period, the subject closed his eyes, keeping the prisms on. For the body larget group, the experimenter removed the dowel and inserted the subject's index finger; for the visual target group. the experimenter left the dowel in place. In either case. the experimenter removed the barrier which prevented the subject from 
reishing the dowel with his pretest pointing. The subject then opened his eyes and in the body target group pointed to his index finger. and in the visual target group pointed to the dowel. In neither group could the subject see his pointing hand, so that feedback consisted only of touching or failing to touch the target.

Posttest. Following the exposure period, the subject again closed his eyes for a few seconds. keeping the prisms on. For subjects in the visual target condition, the barrier which prevented them from reaching the target was reinserted. For subjects in the body target condition, the left index tinger was removed. the dowel reinserted, and the barricr also reinserted. Upon the experimenter's signal, the subject opened his eyes and the pretest measurements were repeated.

\section{Subjects}

Two groups of subjects were used. The subjects consisted of graduate and undergraduate students at the authors' university. Fifteen of the subjects were females, and the remaining 25 were males. All the subjects were right handed. None had any visual defects other than wearing corrective lenses.

\section{RESULTS}

The experiment had the form of a two-factor experiment. The two factors were pointing hand (left and right) and target condition (body and visual). There were repeated measures on the factor of hand. In addition, there were two different dependent variables. The first was the difference between preand posttest straight-ahead pointing; the second was the difference between pre- and posttest target pointing. These will be referred to as the straight-ahead shifts and the target-pointing shifts, respectively. These shifts will be referred to as adaptive when they are in the direction of prismatic displacement.

The straight-ahead shifts were first analyzed. None of the straight-ahead shifts was significantly different from zero. In addition, an analysis of variance was performed for the straight-ahead shifts, using the procedure described by Myers (1966, p. 181). Neither of the two main effects was significant, nor was the interaction between them, and so no further analysis was done on the data.

The same analysis of variance was performed for the target-pointing shifts. There was no main effect of hand, $F(1.38)=0.03$, and no main effect of target condition, $F(1.38)=1.00$. However, there was a signiticant $H$ and by Target interaction, $F(1.38)=$ 22.7, $p<.01$. The mean target-pointing shift in degrees for each hand, in each target condition, is shown in Figure 1. All the pointing shifts shown in Figure 1 are in the adaptive direction, and their value significantly differs from zero at the 0.01 level, one-tailed.

The interaction between hand and target condition is crossed, as Figure 1 shows. In order to analyze it further, we examined the simple effect of target condition in each hand condition, since this bears most directly on our experimental hypothesis. For the right, pointing, hand, there is significantly more

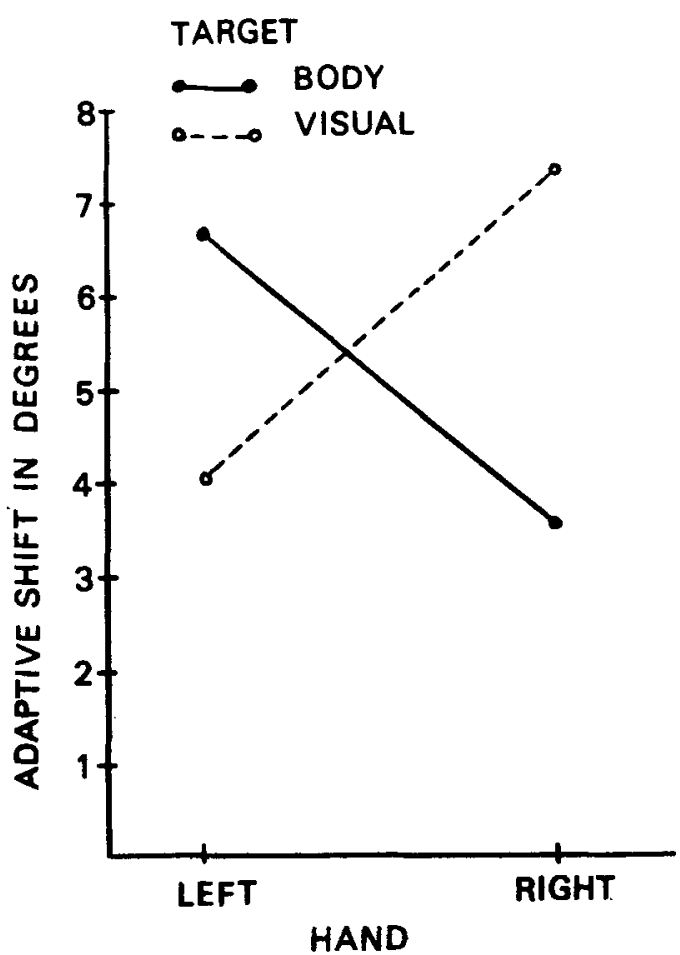

Figure 1. Mean pointing shift in degrees for left and right hands in the body target and visual target conditions.

pointing shift in the visual target condition than in the body target condition, $\mathrm{t}(38)=2.42, \mathrm{p}<.05$, one-tailed. For the left, nonpointing, hand, there is significantly more pointing shift in the body target condition than in the visual target condition, $\mathrm{t}(38)=$ $1.79, p<.01$, one-tailed.

We also analyzed the simple effect of hand in each target condition. In the body target condition, the left hand showed more adaptation than the right hand, $t(19)=2.24, p<.05$, one-tailed. For the visual target condition. the right hand showed more adaptation than the left hand, $t(19)=2.60, p<.01$, one-tailed.

\section{DISCUSSION}

The results confirm the predictions made in the introduction. That is, when the crossed interaction is broken down into simple effects, there is, as predicted, more adaptation for the right hand in the visual target condition than in the body target condition. The subsidiary prediction is also confirmed, namely that there is, as predicted, more adaptation for the left hand in the body target condition than there is in the visual target condition. Since both of these predictions, and particularly the first, follow from the hypothesized assimilation tendency between the right and left hands, we take this as evidence for the hypothesis. This assimilation tendency may be responsible for the result. reported 
by Efstathiou, Bauer. Greene, and Held (1967), that measured adaptive shifts are smaller when the unexposed hand serves as a test target to measure adaptation than when a visual target is used.

Several questions may arise in interpreting the difference between the groups, to which we now turn. For one thing, the visual target was physically straight ahead. and therefore appeared to one side when viewed through the prism. This visual asymmetry is known to affect visual direction. This effect. however, is present in both the visual target and body target conditions, and so does not affect the comparison between the groups. It might be argued, however, that the two groups did not point the same way, since the targets were different. Our response to this is that the movements were the same, namely ballistic movements directed to the target, and the effect of the training procedure was the same, that is, the subjects eventually hit the target. The visual condition was, admittedly, a bit unusual in that the subject could not see their pointing hands and hence received no visual intomation about how far they were off target, but this is true also of the body target condition.

We can only be speculative about the nature of the adaptation found in the present study. It would seem not to be proprioceptive, for no changes were found when pointing straight ahead was used as a dependent variable, and this has been assumed to be a measure of proprioceptive change (Harris. 1965). However, "straight ahead" may mean different things to different people (Templeton et al., 1974), so the measure is somewhat unreliable and it is possible that some proprioceptive change did occur. The adaptive change cannot be entirely visual, since if it were we would expect the pointing behavior of both hands to change equally (Wilkinson, 1971), which was not observed. The most likely hypothesis is that the observed adaptation is some combination of a visual change and what Held calls altered sensorimotor correspondence (Hardt et al., 1971) and Templeton et al. (1974) call a change in coordinators, i.e., a change in the correspondence rule between vision and proprioception which registers the two sources of information as coincident. Since the studies just cited obtained evidence for such a change, we feel that it is legitimate to postulate it here, as well. Of course, further studies investigating different measures of proprioceptive change, and measuring visual change directly, would shed light on these issues.

We do not know why so little proprioceptive change was found and so large an amount of visual-proprioceptive coordinator change. Our best guess is that the conditions of our experiment, in which the subject never saw his pointing hand, but was exposed to a discrepancy between the position of a target registered visually and registered proprioceptively facilitated the coordinator change. The logic behind this guess is that such information is sufficient to establish that vision and proprioception are not in coincidence, but not sufficient to establish which is urong.

The proposed proprioceptive-proprioceptive assimilation tendency has so far been demonstrated through its effect on perceptual adaptation. It may, however, play a positive role in normal orientation behavior. It is possible, for example, that local proprioceptive information begins to drift from indicating the true value. How are such drifts corrected? One mechanism may be sight of the limb in question, as has been suggested by many authors in this area (e.g.. Harris, 1965; Held, 1961). However, the assimilation tendency postulated here might also do the job. In this view, every time one body part touches another body part both parts come to agree on the spatial position that each occupies. A similar process occurring throughout the course of development would assure that the various parts of the body stay in spatial harmony with each other despite the fact that the body parts continually grow.

\section{REFERENCES}

Efstathiou, A., Bauer. J., Greene, M., \& Held, R. Altered reaching following adaptation to optical displacement of the hand. Journal of Experimental Psychology, 1967, 73, 113-120.

hard, M. E., Held, R., \& Steinbach, M. J. Adaptation to displaced vision: A change in the central control of sensori-motor coordination. Journal of Experimental Psychology. 1971, 89, 229-239.

Harris, C. S. Perceptual adaptation to inverted, reversed, and displaced vision. Psychological Review, 1965, 72, 419-444.

HELD, R. Exposure-history as a factor in maintaining stability of perception and coordination. Journal of Nervous and Mental Disease, 1961, 132. 26-32.

MOUlden. B. Adaptation to displaced vision: Reafference is a special case of the cue-discrepancy hypothesis. Quarterly Journal of Experimental Psychology, 1971, 23, 113-117.

MYERS. J. L. Fundamentals of experimental design. Boston: Allyn \& Bacon. 1966.

Templeton, W. B.. Howard, I. P., \& Wilkinson, D. A. Additivity of components of prismatic adaptation. Perception \& Psychophysics, 1974, 15, 249-257.

Wilkinson. D. A. Visual-motor control loop: A linear system? Journal of Experimental Psychology, 1971, 89, 250-257.

W ALLACH, H. Informational discrepancy as a basis of perceptual adaptation. In S. J. Freedman (Ed.), The neuropsychology of spatially oriented behavior. Homewood, Ill: Dorsey Press. 1968.

(Received for publication November 25, 1974; revision received May 1,1975 .) 